\title{
FIXED POINTS AND ITERATION HOMOTOPIES
}

FRANCIS J. PAPP

(Received 30 July 1969)

Communicated by B. Mond

Suppose that $\Phi$ is a topological space equipped with a Hausdorff topology and that $T$ is a continuous function mapping $\Phi$ into $\Phi$. We discuss the existence and uniqueness of fixed points of $T$ and the convergence of the Picard sequence of iterates, from the viewpoint of the existence of a homotopy with special properties.

Definition 1. The mapping $H_{T}(\phi, t)$ of $\Phi \times[0,1]$ to $\Phi$ will be called an iteration homotopy for $T$ if

i) $H_{Y}(\phi, t)$ is a homotopy

ii) there exists a strictly decreasing sequence $\left\{n_{s} \mid s=1,2, \cdots\right\}$ of points in $[0,1]$ for which:

a) $n_{\mathbf{1}}=1$

b) $H_{T}\left(\phi, n_{s}\right)=T^{s} \phi$, where $T^{s} \phi=T\left(T^{s-1} \phi\right)$, $s=2,3, \cdots$

c) $\lim _{s \rightarrow \infty} n_{s}=0$.

THEOREM 2. A function $T$ has at most one fixed point if

i) an iteration homotopy for $T$ exists, and

ii) $H_{T}(\phi, 0)$ has at most one fixed point.

Proof. $T$ is necessarily continuous, since $H_{T}(\phi, 1)=T$ and $H_{T}(\phi, t)$ is a homotopy.

Suppose $\phi$ and $\psi$ are distinct fixed points of $T$. In this case, $T^{k} \phi=\phi$ and $T^{k} \psi=\psi$ for all $k=1,2, \cdots$. But then $\phi=H_{T}\left(\phi, n_{k}\right)$ and $\psi=H_{T}\left(\psi, n_{k}\right)$ for all $k$, and so, from the assumed continuity of $H_{T}$,

$$
\phi=\lim _{k \rightarrow \infty} H_{T}\left(\phi, n_{k}\right)=H_{T}(\phi, 0) .
$$

Likewise, $\psi=H_{T}(\psi, 0)$.

CoROllaRY 3. If for some element $\psi$ of $\Phi, H_{T}(\phi, 0)=\psi$ for all $\phi \in \Phi$, then: 
i) Thas at most one fixed point,

ii) either the fixed point is $\psi$, or there is no fixed point,

iii) the sequence of iterates converges for all $\phi \in \Phi$, and the limit is independent of the initial point $\phi$.

COROLlaRy 4. For some positive integer $n_{0}$ let $T^{n_{0}}$ satisfy the hypotheses of the theorem. If $T$ is continuous, then $T$ has at most one fixed point.

Proof. Every fixed point of $T$ is a fixed point of $T^{n o}$.

\section{EXAMPLE 5.}

i) If $\Phi$ represents the real number system and $T_{x}=e^{-x}$, Corollary 3 applies.

ii) If $\Phi=L_{2}\left(R^{\#}\right)$ and $T \phi(x)=f(x)+\lambda \int_{0}^{x} e^{(x-y)} \phi(y) d y$, the following mapping is an iteration homotopy for $T$ for which $H_{T}(\phi, 0)$ is a constant function on $\Phi$ :

$$
H_{T}(\phi, t)=\left[n^{2} t+n t-n\right] T^{n} \phi+\left[1-n^{2} t-n t+n\right] T^{n+1} \phi
$$

for $t \in[1 /(n+1), 1 / n], n=1,2, \cdots$.

It is not difficult to show that

$$
H_{T}(\phi, 0)=f(x)+\int_{0}^{x} f(y) e^{(1+\lambda)(x-y)} d y .
$$

Finally,

i) $H_{T}(\phi, 0)$ is independent of $\phi$

ii) $K \phi=H_{T}(\phi, 0)$ has at most one fixed point as it is a constant function, i.e. $K\left(H_{T}(\phi, 0)\right)=H_{T}\left(H_{T}(\phi, 0), 0\right)=H_{T}(\phi, 0)$, and

iii) by Corollary $3, T=H(\cdot, 1)$ has at most one fixed point. It is easily verified that

$$
H_{T}\left(H_{T}(\phi, 0), 1\right)=T\left(H_{T}(\phi, 0)\right)=H_{T}(\phi, 0) .
$$

In certain instances it may be more convenient to consider a modification of the criginal function, and to obtain first the fixed points of the modified function. To yield information regarding the fixed points of the original mapping, the modification procedure must, in some sense, be reversible.

Examples of the procedure to be discussed below can be found in S. C. Chu and J. B. Diaz [1].

The use of iteration homotopies permits the following generalizations of the theorems of [1], in the sense that the space is not assumed to be normed.

THEOREM 6. If $T, K, K^{-1}$ are continuous members of $\Phi^{\Phi}$ for which:

i) $K K^{-1}$ is the identity map on $\Phi$,

ii) there exists an iteration homotopy $H$ for the mapping $K^{-1} T K$, and 
iii) $H(\phi, 0)$ has at most cne fixed point, then $T$ has at most one fixed point.

Proof. By Theorem 2, $K^{-1} T K$ has at most one fixed point. Moreover, corresponding to each fixed point of $K^{-1} T K$ there is a fixed point of $T$ and conversely.

COROLLARY 7. If $H$ denotes the iteration homotopy for $K^{-1}$ TK and $H(\phi, 0)=\psi$ for all $\phi \in \Phi$, then either the unique fixed point of $T$ is $K \psi$, or $T$ has no fixed point.

Proof. If $H(\phi, 0)=\psi$ for all $\phi \in \Phi$, the unique fixed point of $K^{-1} T K$ must be $\psi$, if there is one. But then, if $K^{-1} T K \psi=\psi, T K \psi=K K^{-1} T K \psi=K \psi$.

The theorem and corollary which follow show that it suffices that some iterate of the given function be amenable to modification of the type indicated above.

THEOREM 8. If $T, K, K^{-1}$ are continuous members of $\Phi^{\Phi}$ and $n_{0}$ is a positive integer for which

i) $K K^{-1}$ is the identity map on $\Phi$

ii) there exists an iteration homotopy $H$ for the mapping $K^{-1} T^{n_{0}} K$, and

iii) $H(\phi, 0)$ has at most one fixed point,

then $T$ has at most one fixed point.

Proof. By Theorem 2 applied to the mapping $K^{-1} T^{n_{0}} K, K^{-1} T^{n_{0}} K$ has at most one fixed point. To every fixed point of $K^{-1} T^{n_{0}} K$ corresponds a fixed point of $T^{n_{0}}$, and conversely. Finally, every fixed point of $T$ is a fixed point of $T^{n_{0}}$.

COROLlaRY 9. If $H$ denotes the iteration homotopy for $K^{-1} T^{n_{0}} K$, and $H(\phi, 0)=\psi$ for all $\psi \in \Phi$, then either the unique fixed point of $T$ is $K \psi$, or T has no fixed point.

Proof. As in the proof of Corollary 7, either $T^{n_{0}} K \psi=K \psi$, or $T^{n_{0}}$ has no fixed point. If $T$ has a fixed point, $\phi$, say, then $T^{k} \phi=\phi$ for all $k$, and, in particular, $k=n_{0}$. However, the fixed point of $T^{n_{0}}$ is unique and hence $\phi=K \psi$.

If $\Phi$ is a Banach space, and if $T$ is a contraction, the classical theorem of Banach $[2 ; 78)$ insures that $T$ has at most one fixed point, and that $T^{n}$ converges to the unique flxed point, $\psi$, say, for any choice of $\phi$. The Banach contraction principle states that, for any iteration homotopy for $T, H_{T}(\phi, t)$, say, then $H_{T}(\phi, 0)$ $=\psi$ for all $\phi \in \Phi$, and $H_{T}(\psi, 1)=\psi$.

An iteration homotopy for a given contraction may be constructed as in Example 5 (ii); namely, set

$$
H_{T}(\phi, t)=\left[n^{2} t+n t-n\right] T^{n} \phi+\left[1-n^{2} t-n t+n\right] T^{n+1} \phi
$$

for $t \in[1 /(n+1), 1 / n], n=1,2, \cdots$. 
Finally, if $T$ is not a contraction, then it may be possible to choose a map $K$ such that $K^{-1} T K$ or $K^{-1} T^{n_{0}} K$ is a contraction. If this is possible, the Banach contraction principle, in connection with the theorems of Chu and Diaz, give the desired analogous information about the fixed point of $T$, for the case in which $\Phi$ is a Banach space.

\section{References}

[1] S. C. Chu and J. B. Diaz, 'A Fixed Point Theorem for 'In the Large' Application of the Contraction Principle', Atti della Accademia delle Scienze di Torino, Vol. XCIX, (196465), 351-363.

[2] E. Hewitt and K. Stromberg, Real and Abstract Analysis (New York: Springer-Verlag; 1965).

Department of Mathematics

University of Lethbridge

Lethbridge, Alberta, Canada 